\title{
Romosozumab and antiresorptive treatment: the importance of treatment sequence
}

\author{
Felicia Cosman ${ }^{1}$ (1) - David L. Kendler ${ }^{2} \cdot$ Bente L. Langdahl $^{3} \cdot$ Benjamin Z. Leder ${ }^{4} \cdot$ E. Michael Lewiecki $^{5}$. \\ Akimitsu Miyauchi $^{6} \cdot$ Maria Rojeski $^{7} \cdot$ Michele McDermott $^{7} \cdot$ Mary K. Oates $^{7} \cdot$ Cassandra E. Milmont $^{7}$. \\ Cesar Libanati ${ }^{8} \cdot$ Serge Ferrari $^{9}$
}

Received: 21 April 2021 / Accepted: 23 September 2021 / Published online: 15 February 2022

(c) The Author(s) 2022

\begin{abstract}
Summary To evaluate whether treatment sequence affects romosozumab response, this analysis reviewed studies where romosozumab was administered before or following an antiresorptive (alendronate or denosumab). Initial treatment with romosozumab followed by an antiresorptive resulted in larger increases in bone mineral density of both hip and spine compared with the reverse sequence.

Introduction Teriparatide followed by an antiresorptive increases bone mineral density (BMD) more than using an antiresorptive first. To evaluate whether treatment sequence affects romosozumab response, we reviewed randomized clinical trials where romosozumab was administered before (ARCH, FRAME) or following (STRUCTURE, Phase 2 extension) an antiresorptive (alendronate or denosumab, respectively).

Methods We evaluated BMD percentage change for total hip (TH) and lumbar spine (LS) and response rates (BMD gains $\geq 3 \%$ and $\geq 6 \%$ ) at years 1 and 2 (except STRUCTURE with only 1-year data available).

Results With 1-year romosozumab initial therapy in ARCH and FRAME, TH BMD increased 6.2\% and 6.0\%, and LS BMD increased $13.7 \%$ and $13.1 \%$, respectively. When romosozumab was administered for 1 year after alendronate (STRUCTURE) or denosumab (Phase 2 extension), TH BMD increased 2.9\% and 0.9\%, respectively, and LS BMD increased 9.8\% and 5.3\%, respectively. Over 2 years, TH and LS BMD increased $7.1 \%$ and $15.2 \%$ with romosozumab/alendronate, $8.5 \%$ and $16.6 \%$ with romosozumab/denosumab, and 3.8\% and $11.5 \%$ with denosumab/romosozumab, respectively. A greater proportion of patients achieved BMD gains $\geq 6 \%$ when romosozumab was used first, particularly for $\mathrm{TH}$, versus the reverse sequence $(69 \%$ after romosozumab/denosumab; $15 \%$ after denosumab/romosozumab).

Conclusion In this study, larger mean BMD increases and greater BMD responder rates were achieved when romosozumab was used before, versus after, an antiresorptive agent. Since BMD on treatment is a strong surrogate for bone strength and fracture risk, this analysis supports the thesis that initial treatment with romosozumab followed by an antiresorptive will result in greater efficacy versus the reverse sequence.
\end{abstract}

Keywords Anabolic $\cdot$ Antiresorptive $\cdot$ Romosozumab $\cdot$ Teriparatide $\cdot$ Treatment sequence

Felicia Cosman

fc14@cumc.columbia.edu

1 College of Physicians and Surgeons, Columbia University, $630 \mathrm{~W} 168$ th St, New York, NY 10032, USA

2 University of British Columbia, Vancouver, BC, Canada

3 Aarhus University Hospital, Aarhus, Denmark

4 Massachusetts General Hospital and Harvard Medical School, Boston, MA, USA
5 New Mexico Clinical Research \& Osteoporosis Center, Albuquerque, NM, USA

6 Miyauchi Medical Center, Osaka, Japan

7 Amgen Inc., Thousand Oaks, CA, USA

8 UCB Pharma, Brussels, Belgium

9 Geneva University Hospital, Geneva, Switzerland 


\section{Introduction}

Over the past two decades, studies comparing bone-forming with antiresorptive agents in head-to-head trials have shown significantly lower incidence of fractures with bone-forming agents [1-5]. These findings have led to recommendations to consider treatment with bone-forming agents as initial therapy in patients at very high risk for fracture, particularly those who require rapid fracture risk reduction [6, 7]. Most participants in these trials have been treatment naïve or have had minimal recent exposure to osteoporosis treatment. Fracture outcomes are not available from smaller studies designed to evaluate the BMD effects of teriparatide or romosozumab following treatment with bisphosphonates [8-13] or denosumab [13, 14].

In a large meta-regression analysis of 38 randomized clinical trials involving 19 different therapeutic agents for osteoporosis, the magnitude of total hip BMD gain has emerged as a relevant surrogate endpoint for estimating fracture risk reduction [15]. In that analysis, treatments producing a total hip BMD gain of $2 \%$ would be expected to reduce hip fracture occurrence by $16 \%$, whereas treatments producing a total hip BMD gain of $6 \%$ would be expected to reduce hip fracture incidence by $40 \%$ [15]. Furthermore, in individual patients, the total hip BMD level attained during or after treatment with alendronate, zoledronic acid, denosumab, or romosozumab is a major predictor of subsequent absolute risk of fracture and accordingly could serve as a treatment target for osteoporosis therapy [16-20].

Past treatment sequence studies indicate that starting with teriparatide and then transitioning to an antiresorptive agent increases BMD more than the reverse sequence, with the most prominent differences seen in the hip region [14, 21]. In fact, total hip BMD declines and remains below baseline for at least 12 months in patients who transition from bisphosphonates to teriparatide and declines prominently and remains below baseline for up to 24 months in patients who switch from denosumab to teriparatide [14, 21]. Data evaluating the sequence of a bisphosphonate or denosumab followed by abaloparatide are not available, although the sequence of abaloparatide first, followed by alendronate, produces substantial benefits to hip BMD [22, 23].

Romosozumab (EVENITY ${ }^{\circledR}$ [romosozumab-aqqg in the USA]) [24] is a bone-forming agent with the dual effect of increasing bone formation and decreasing bone resorption $[25,26]$. When romosozumab is given as the initial therapy, marked increases in BMD have been observed, with further BMD increases upon transition to an antiresorptive agent as reported in the FRAME and $\mathrm{ARCH}$ randomized controlled trials (RCTs) [5, 27]. Two additional RCTs provide data for evaluation of bone turnover markers and
BMD effects when antiresorptive agents are given first, followed by romosozumab [12, 28].

In this analysis, we reviewed data from the four RCTs that evaluated treatment sequences of romosozumab administered before or following an antiresorptive agent, either alendronate or denosumab [5, 12, 27-29]. We evaluated changes in total hip and lumbar spine BMD, the proportions of patients who achieved BMD gains $\geq 3 \%$ and $\geq 6 \%$ at the total hip and lumbar spine, and the profile of changes in levels of the bone formation marker procollagen type I N-terminal propeptide (PINP) and the bone resorption marker $\beta$-isomer of the C-terminal telopeptide of type I collagen $(\beta$-CTX) after 12 and 24 months of treatment with the different treatment sequences.

\section{Materials and methods}

\section{Studies included in analysis and outcomes}

Data from four separate RCTs were analyzed for changes from baseline in BMD, PINP, and $\beta$-CTX and response rates (BMD gains $\geq 3 \%$ and $\geq 6 \%$ ): (1) the $\underline{\text { Active-cont }}$ Rolled fraCture study in postmenopausal women with osteoporosis at High risk (ARCH; NCT01631214) [5], (2) the FRActure study in postmenopausal women with ostEoporosis (FRAME; NCT01575834) [27], (3) the STudy evaluating effect of $\underline{\mathbf{R}}$ omosoz Umab compared with teriparatide in postmenopaUsal women with osteoporosis at high risk for fracture p $\underline{\mathbf{R}}$ eviously treated with bisphosphonat $\underline{\mathbf{E}}$ therapy (STRUCTURE; NCT01796301) [12], and (4) the romosozumab Phase 2 dose-finding study extension (Phase 2 extension; NCT00896532) [28, 29]. The study designs of the four RCTs are illustrated in Fig. 1.

ARCH [5] had randomized 4093 women aged 55 to 90 years with osteoporosis and a prior fragility fracture to receive double-blinded subcutaneous romosozumab $210 \mathrm{mg}$ once monthly or weekly oral alendronate $70 \mathrm{mg}$ for 12 months, followed by open-label weekly oral alendronate $70 \mathrm{mg}$ in both groups (Fig. 1). In this analysis, we focus on the study arm that received romosozumab followed by alendronate. BMD at the lumbar spine and total hip was measured at baseline and every 12 months. Mean percentage changes from baseline in BMD are presented for the romosozumab arm for patients who had a baseline measurement and $\geq 1$ postbaseline measurement ( $n=1826$ for total hip BMD; $n=1750$ for lumbar spine BMD) [5]. Serum PINP and $\beta$-CTX levels were assessed in 137 women who had enrolled in the biomarker substudy at baseline (before the first romosozumab dose) and at months 1, 3, 6, 9, 12, 15, 18, and 24. Median absolute changes from baseline (pre-dose) in PINP and $\beta$-CTX levels are presented through month 24 . 


\section{Romosozumab in Treatment-naïve Women}

\section{a. $\mathrm{ARCH}$}

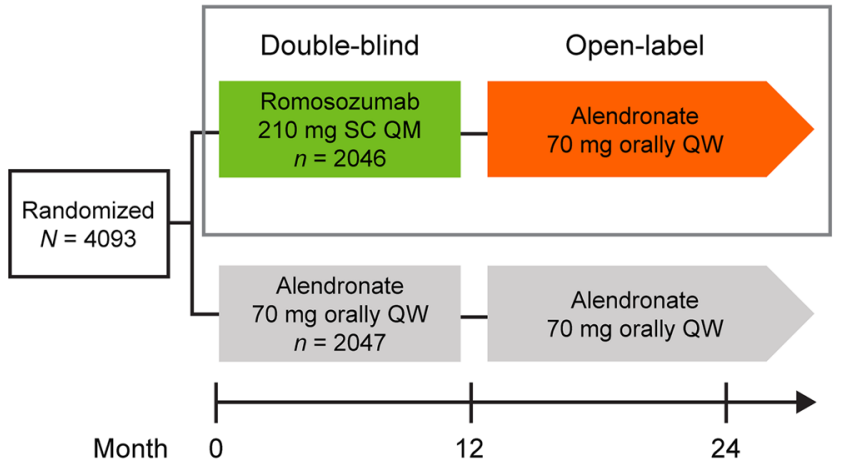

\section{b. FRAME}

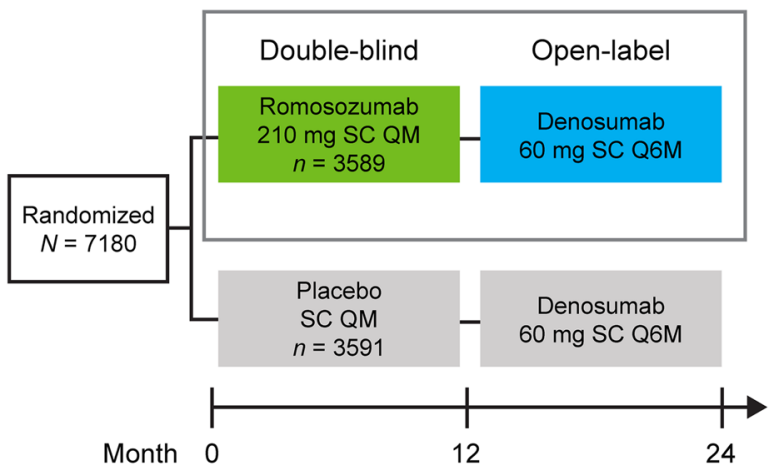

Romosozumab in Women Treated With Antiresorptive Agents First

\section{c. STRUCTURE}

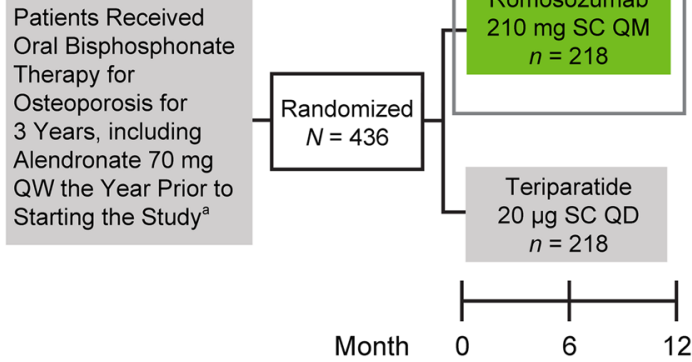

Fig. 1 Study designs for ARCH (a), FRAME (b), STRUCTURE (c), and Phase 2 extension (d). For each study, the gray box depicts the study arm that was not included in this analysis. $N=$ number of patients in the study. $n=$ number of patients in each study arm. ${ }^{\mathrm{a}} \mathrm{Pa}-$ tients received alendronate in the 1 year immediately before screen-

FRAME [27] had randomized 7180 women, aged 55 to 90 years, with a T-score of -2.5 to -3.5 at the total hip or femoral neck, to receive double-blinded subcutaneous romosozumab $210 \mathrm{mg}$ or placebo monthly for 12 months, after which both groups received open-label subcutaneous denosumab $60 \mathrm{mg}$ every 6 months for an additional 12 months (Fig. 1). In this analysis, we focus on the study arm that received romosozumab followed by denosumab. BMD at the lumbar spine and proximal femur was measured at baseline and at months 12 and 24. Mean percentage changes from baseline in BMD are presented for the 3238 patients from the romosozumab arm who had a baseline measurement and $\geq 1$ postbaseline assessment. Serum levels of PINP and $\beta$-CTX were assessed in 62 women who had enrolled in the biomarker study at baseline (before the first romosozumab dose) and months 1, 3, 6, 9, 12, 13, 18, and 24. Median absolute changes from baseline (pre-dose) d. Phase 2 Extension ${ }^{b}$

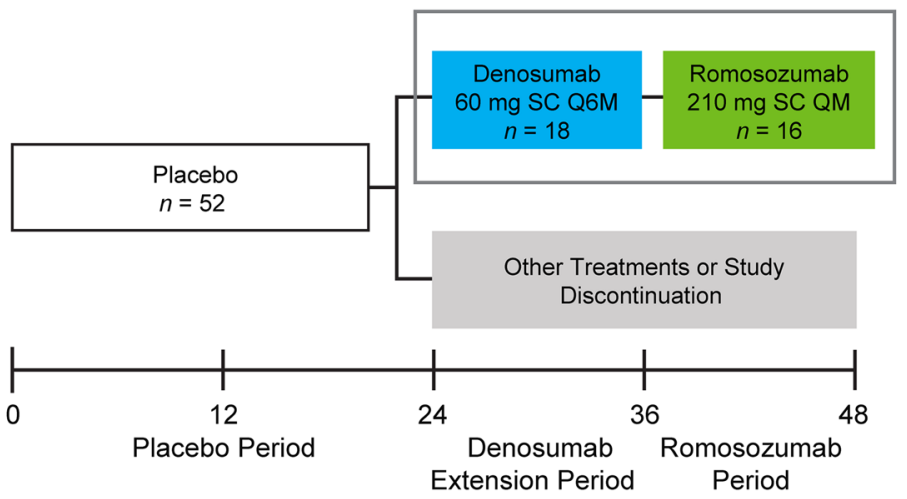

ing. ${ }^{\mathrm{b}}$ Of the 52 women initially randomized to placebo from months $0-24,18$ were rerandomized to denosumab and 34 to other treatments or discontinued study. $Q 6 M$ every 6 months, $Q D$ daily, $Q M$ monthly, $Q W$ weekly, $S C$ subcutaneous

in PINP and $\beta$-CTX levels are presented through month 24.

STRUCTURE [12] had enrolled 436 women aged 55 to 90 years, with a history of nonvertebral fracture after the age of 50 years or a vertebral fracture and T-score of $\leq-2.5$ at the total hip, lumbar spine, or femoral neck who had received oral bisphosphonate therapy for $\geq 3$ years and oral weekly alendronate ( $70 \mathrm{mg}$ or equivalent) for $\geq 1$ year prior to screening. Women were randomly assigned to receive open-label subcutaneous romosozumab $210 \mathrm{mg}$ monthly (218 patients) or daily subcutaneous teriparatide $20 \mu \mathrm{g}$ (218 patients) for 12 months (Fig. 1). In this analysis, we focus on the arm that received romosozumab after alendronate. $\mathrm{BMD}$ at the lumbar spine and proximal femur was measured at baseline and at months 6 and 12. Percentage changes from baseline in BMD are presented for the 197 patients who received romosozumab and had BMD measurements 
at specified timepoints. Serum levels of PINP and $\beta$-CTX were assessed in all enrolled women at baseline (before the first romosozumab dose) and months 1, 3, 6, 9, and 12 . Median absolute changes from baseline (pre-dose) in PINP and $\beta$-CTX levels are presented through month 12 for the 215 patients who received romosozumab and had PINP and $\beta$-CTX assessments at specified timepoints.

The romosozumab Phase 2 dose-finding study and its extensions $[25,28,30]$ had randomized 419 women 55 to 85 years old with a low BMD (T-score of $\leq-2.0$ and $\geq-3.5$ at the lumbar spine, total hip, or femoral neck) into multiple arms and interventions over a 6-year period. In this analysis, we focus on the subset of patients $(n=16)$ who were randomized to placebo for 24 months, followed by denosumab for 12 months, and then received romosozumab $210 \mathrm{mg}$ for an additional 12 months [28, 29] (Fig. 1). For this analysis, percentage changes from baseline in BMD were assessed during the 12 months of romosozumab administration (months 36-48) following denosumab treatment (months 24-36) and cumulatively during 1 year of denosumab followed by 1 year of romosozumab from months 24-48. Serum levels of PINP and $\beta$-CTX were assessed at the start of the romosozumab period (at month 36) as the baseline/ month 0 (before the first romosozumab dose) and at months $1,3,6,9$, and 12 during the romosozumab period. Median absolute changes in PINP and $\beta$-CTX levels from the month 36 baseline/month 0 (pre-dose) of the romosozumab period are presented up to 1 year of the romosozumab administration period.

Across the four studies, BMD at the lumbar spine and proximal femur had been evaluated by dual-energy x-ray absorptiometry (DXA; Lunar, GE Medical Systems, Madison, WI, USA or Hologic, Hologic Inc., Bedford, MA, USA) at baseline and at the specified times for each study. BioClinica (previously known as Synarc, Newark, CA, USA) analyzed the scans and provided quality control of individual scans and densitometers. Blood had been collected and analyzed by contract research laboratories to assess levels of PINP and $\beta$-CTX.

For the four studies, a responder analysis assessed the proportion of patients with a BMD percent change from baseline $\geq 3 \%$ and $\geq 6 \%$ (responders) at both the total hip and lumbar spine at months 12 and 24 . The $\geq 3 \%$ and $\geq 6 \%$ cutoff points are consistent with cutoff points that have been previously published, with the $\geq 3 \%$ cutoff for response versus nonresponse representing the approximate least significant change for serial BMD measurements by DXA [31].

\section{Statistical analysis}

Percentage changes from baseline in BMD across the four studies were assessed using an analysis of covariance (ANCOVA) model adjusting for baseline covariates in
FRAME [27], using a repeated measures model adjusting for baseline covariates in ARCH [5] and STRUCTURE [12], and as summary statistics in the Phase 2 extension $[28,29]$. Results are presented as LS means and 95\% CI for ARCH, FRAME, and STRUCTURE and means and 95\% CI based on summary statistics for the Phase 2 extension. Comparisons across studies are expressed descriptively only. For the responder analysis, data assessed were based on last observation carried forward for ARCH and FRAME, observed data for STRUCTURE, and observed data for the Phase 2 extension. Serum levels of PINP and $\beta$-CTX and absolute changes from baseline are presented. Percentage changes from baseline in PINP and $\beta$-CTX across the four studies were assessed by descriptive statistics and are presented as medians and interquartile ranges.

\section{Results}

\section{Baseline characteristics}

Baseline characteristics of patients from the four studies included in this analysis are summarized in Table 1. This includes 2046 patients enrolled in the romosozumab-toalendronate group in ARCH [5]; 3589 patients enrolled in the romosozumab-to-denosumab group in FRAME [27]; 218 patients enrolled in the romosozumab group after stopping alendronate in STRUCTURE [12]; and 16 patients who were initially randomized to placebo for 24 months, followed by denosumab for 12 months, and then transitioned to romosozumab for an additional 12 months in the romosozumab Phase 2 extension [28, 29].

Baseline demographic and clinical characteristics varied across the four studies (Table 1). In ARCH [5], 96\% of women had a prevalent vertebral fracture, and 38\% had a nonvertebral fracture at baseline. In STRUCTURE [12], all women had prevalent vertebral and/or nonvertebral fractures prior to enrollment. In FRAME [27], 19\% of patients had a prior vertebral fracture and $22 \%$ had a previous nonvertebral fracture at baseline. Mean ages were oldest in ARCH (74 years), similar in FRAME and STRU CTURE (71 and 72 years, respectively) and youngest in the Phase 2 extension (67 years). Furthermore, consistent with recruitment criteria, mean baseline T-scores were similar in ARCH, FRAME, and STRUCTURE, but higher in the Phase 2 extension. Baseline levels of bone turnover markers reflected untreated postmenopausal status in ARCH and FRAME [5, 27]. Baseline median levels of both PINP and $\beta$-CTX were low in STRUCTURE and the Phase 2 extension, consistent with prior alendronate and denosumab treatment, respectively [12, 28, 29]. 
Table 1 Baseline characteristics of patients included in the four studies

\begin{tabular}{|c|c|c|c|c|}
\hline & $\mathrm{ARCH}$ & FRAME & STRUCTURE $^{\mathrm{a}}$ & $\begin{array}{l}\text { Phase } 2 \\
\text { Extension }^{\mathrm{b}}\end{array}$ \\
\hline & $\begin{array}{l}\text { Romosozumab to alen- } \\
\text { dronate } \\
n=2046\end{array}$ & $\begin{array}{l}\text { Romosozumab to deno- } \\
\text { sumab } \\
n=3589\end{array}$ & $\begin{array}{l}\text { Alendronate to romo- } \\
\text { sozumab } \\
n=218\end{array}$ & $\begin{array}{l}\text { Denosumab to } \\
\text { romosozumab } \\
n=16\end{array}$ \\
\hline Age, mean (SD), years & $74.4(7.5)$ & $70.9(7.0)$ & $71.8(7.4)$ & $67.1(4.1)$ \\
\hline \multicolumn{5}{|l|}{ BMD T-score, mean (SD) } \\
\hline Lumbar spine & $-2.9(1.3)$ & $-2.7(1.0)$ & $-2.8(1.1)$ & $-2.01(0.5)$ \\
\hline Total hip & $-2.8(0.7)$ & $-2.5(0.5)$ & $-2.3(0.8)$ & $-1.04(0.6)$ \\
\hline Femoral neck & $-2.9(0.5)$ & $-2.8(0.3)$ & $-2.5(0.7)$ & $-1.59(0.5)$ \\
\hline Prevalent vertebral fracture, $n(\%)$ & $1969(96.2)$ & $672(18.7)$ & $218(100)^{\mathrm{c}}$ & ND \\
\hline Previous nonvertebral fracture, $n(\%)$ & $767(37.5)$ & $778(21.7)$ & $218(100)^{\mathrm{c}}$ & ND \\
\hline PINP, median (Q1, Q3), $\mu \mathrm{g} / \mathrm{L}$ & $50.6(37.5,64.7)^{\mathrm{d}}$ & $50.3(36.2,65.9)^{\mathrm{e}}$ & $25.0(18.0,34.0)$ & $17.4(11.2,21.4)$ \\
\hline$\beta$-CTX, median (Q1, Q3), ng/L & $276(166,407)^{\mathrm{d}}$ & $551(338,706)^{\mathrm{e}}$ & $229(150,318)$ & $163(96,268)$ \\
\hline
\end{tabular}

Data analyzed were from four studies: (1) ARCH (NCT01631214), FRAME (NCT01575834), STRUCTURE (NCT01796301), and the Phase 2 extension (NCT00896532)

$n=$ number of patients who received romosozumab in each study

${ }^{\text {a }}$ Patients received oral bisphosphonate therapy for osteoporosis for 3 years, including alendronate for the year prior to starting the study. The median duration of previous oral bisphosphonate use was $6.2( \pm 2.9)$ years, while duration of previous alendronate use was $5.5( \pm 3.2)$ years

${ }^{\mathrm{b}}$ Baseline data shown are for month 36 of the Phase 2 extension when patients transitioned from denosumab to romosozumab; duration of previous denosumab use was 1 year

${ }^{\mathrm{c}}$ All patients had historical fracture (i.e., nonvertebral fractures after age 50 or vertebral fracture), but fractures were self-reported and not confirmed or adjudicated

${ }^{\mathrm{d}}$ Data are for the 137 patients who enrolled in the biomarker substudy, received romosozumab, and had PINP and $\beta$-CTX measurements at baseline and at $\geq 1$ postbaseline visits

${ }^{\mathrm{e}}$ Data are for the patients who enrolled in the biomarker substudy, received romosozumab, and had PINP $(n=62)$ and $\beta$-CTX ( $\left.n=61\right)$ measurements at baseline and at $\geq 1$ postbaseline visits

$\beta$-CTX $\beta$-isomer of the C-terminal telopeptide of type I collagen, BMD bone mineral density, $N D$ not determined, PINP procollagen type I N-terminal propeptide, $Q 1, Q 3$ first and third quartiles, $S D$ standard deviation

\section{BMD gains}

\section{Total hip BMD gains}

Mean percentage changes from baseline in total hip BMD across the four studies are presented in Fig. 2a and b. Mean BMD increased $6.2 \%$ in ARCH and 6.0\% in FRAME with 1 year of romosozumab (Fig. 2a). In STRUCTURE, after patients had switched from alendronate, mean BMD increased $2.9 \%$ with 1 year of romosozumab. In the Phase 2 extension, after 1 year of denosumab treatment, mean BMD increased $0.9 \%$ with romosozumab. With the 2-year sequence, total hip BMD increased a total of $7.1 \%$ in ARCH with romosozumab followed by alendronate and $8.5 \%$ in FRAME with romosozumab followed by denosumab (Fig. 2b). In the Phase 2 extension, the total 2-year gain was $3.8 \%$ when denosumab was followed by romosozumab. Cumulative BMD gains for a 24-month sequence of alendronate followed by romosozumab are not available for STRUCTURE.

\section{Lumbar spine BMD gains}

Mean percentage changes from baseline in lumbar spine BMD across the four studies are presented in Fig. 2c and d. Mean BMD increased $13.7 \%$ in ARCH and $13.1 \%$ in FRAME with 1 year of romosozumab (Fig. 2c). In STRUCTURE, after patients stopped alendronate, mean BMD increased $9.8 \%$ with 1 year of romosozumab. In the Phase 2 extension, after stopping 1 year of denosumab treatment, mean BMD increased $5.3 \%$ with 1 year of romosozumab. With the 2-year sequence, lumbar spine BMD increased a total of $15.2 \%$ in ARCH with romosozumab followed by alendronate and $16.6 \%$ in FRAME with romosozumab followed by denosumab (Fig. 2d). In the Phase 2 extension, the total 2-year BMD gain was $11.5 \%$ with denosumab followed by romosozumab. As already stated, the cumulative BMD gains for a 24-month sequence of alendronate followed by romosozumab are not available for STRUCTURE. 


\section{Total Hip}

\section{a. 1 Year Gains With Romosozumab}

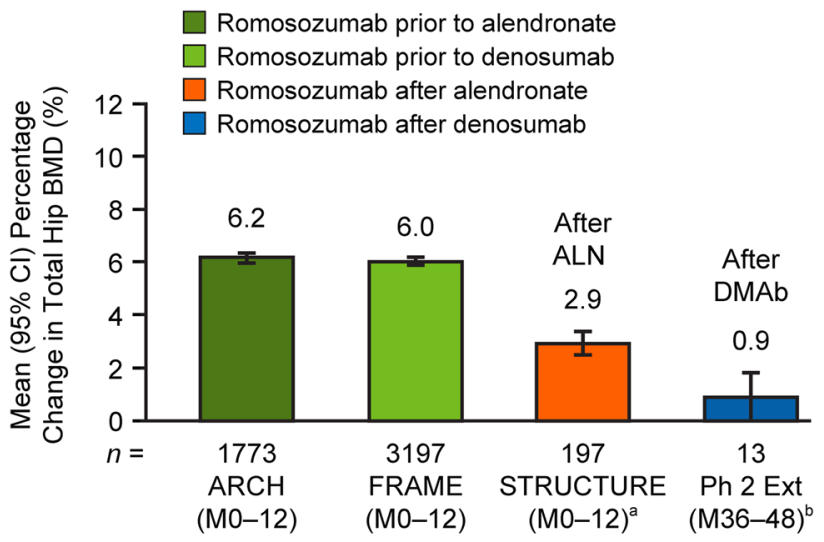

b. Cumulative 2 Year Gains After Sequential Treatment

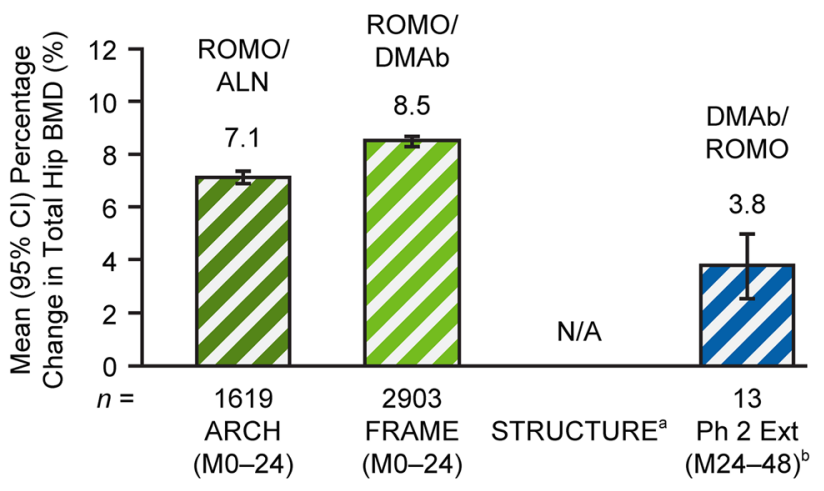

\section{c. 1 Year Gains With Romosozumab}

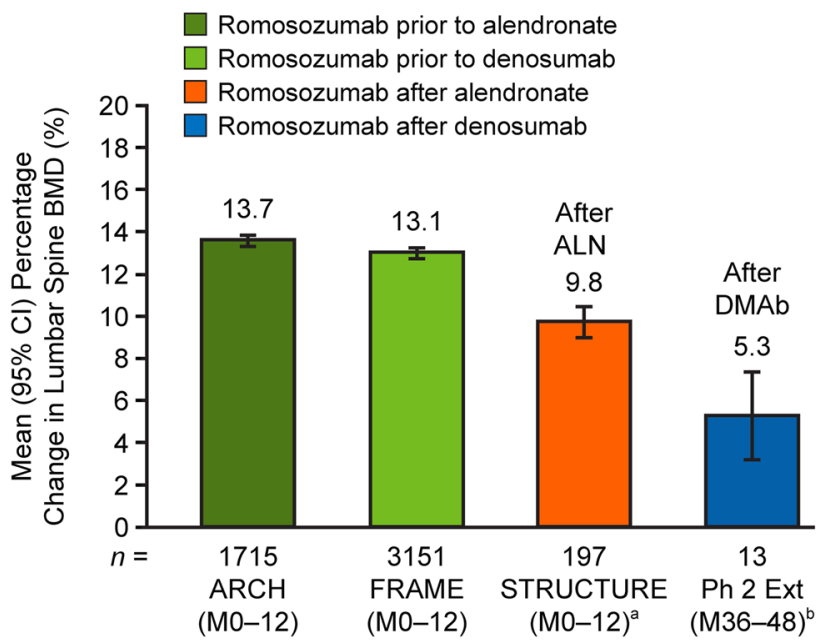

Fig. 2 Mean percentage change from baseline in total hip BMD (a, b) and lumbar spine $(\mathbf{c}, \mathbf{d})$ at 1 year with romosozumab and cumulative 2 years after sequential treatment. Data analyzed were from four studies: ARCH (NCT01631214), FRAME (NCT01575834), STRUCTURE (NCT01796301), and the Phase 2 extension (NCT00896532). $n=$ number of patients who received romosozumab and had total hip or lumbar spine BMD measurements at baseline and at specified timepoints. Percentage changes from baseline were assessed by an ANCOVA model adjusted for baseline covariates in FRAME, a repeated measures model adjusted for baseline covariates in ARCH and STRUCTURE, and as summary statistics in the Phase 2 extension. Least squares means and 95\% CI are shown for ARCH, FRAME, and STRUCTURE, and means

\section{BMD responder analysis}

\section{Total hip BMD responder analysis}

The proportions of patients who achieved BMD gains $\geq 3 \%$ and $\geq 6 \%$ from baseline (responders) at the total hip with 1 year of romosozumab were $74 \%$ and

\section{d. Cumulative 2 Year Gains After Sequential Treatment}

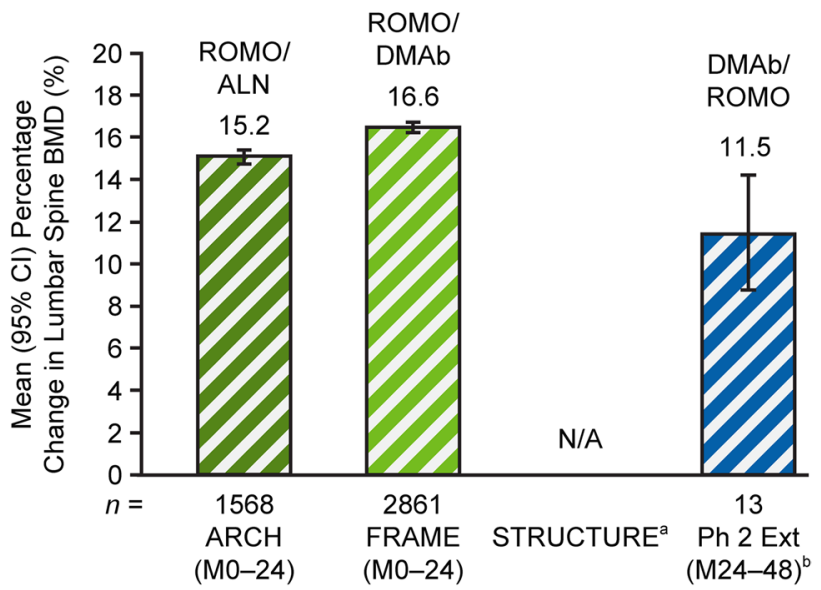

and $95 \%$ CI based on summary statistics are shown for the Phase 2

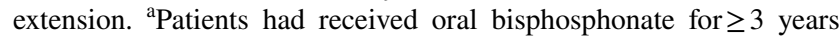
before screening and alendronate $(70 \mathrm{mg} \mathrm{QW})$ for $\geq 1$ year immediately before screening; BMD was not measured in the 1 year of alendronate before romosozumab. ${ }^{b}$ Patients received placebo during months $0-24$, denosumab during months $24-36$, and romosozumab during months 36-48; cumulative gains are relative to the month 24 baseline. $A L N$ alendronate; ANCOVA analysis of covariance; $B M D$ bone mineral density; $C I$ confidence interval; $D M A b$ denosumab; $M$ month; N/A not applicable; Ph 2 Ext Phase 2 Extension; $Q W$ weekly; ROMO romosozumab

$47 \%$, respectively, in $\mathrm{ARCH}$ and were very similar in FRAME (Table 2). In STRUCTURE, after patients had stopped alendronate, the proportion of patients classified as responders to romosozumab was substantially lower for both cutoff points, and in the Phase 2 extension, after completion of 1 year of denosumab, fewer than $10 \%$ of patients achieved the lower cutoff point with 
Table 2 Proportion of patients who achieved BMD percentage change from baseline $\geq 3 \%$ and $\geq 6 \%$ at the total hip and lumbar spine

\begin{tabular}{|c|c|c|c|c|}
\hline & $\begin{array}{l}\text { ARCH } \\
n=2046 \\
n 2 / n 3(\%)\end{array}$ & $\begin{array}{l}\text { FRAME } \\
n=3589 \\
n 2 / n 3(\%)\end{array}$ & $\begin{array}{l}\text { STRUCTURE } \\
n=218 \\
n 2 / n 3(\%)\end{array}$ & $\begin{array}{l}{\text { Phase } 2 \text { Extension }^{\mathrm{a}}} \\
n=16 \\
n 2 / n 3(\%)\end{array}$ \\
\hline & Romosozumab to alendronate & Romosozumab to denosumab & $\begin{array}{l}\text { Alendronate to } \\
\text { romosozumab }\end{array}$ & $\begin{array}{l}\text { Denosumab to } \\
\text { romosozumab }\end{array}$ \\
\hline \multicolumn{5}{|c|}{ Achieved BMD percentage change from baseline $\geq 3 \%$} \\
\hline Total hip & $n 1=1826$ & $n 1=3238$ & $n 1=206$ & $n 1=16$ \\
\hline Month 12 & $1320 / 1781(74.1)$ & $2485 / 3197(77.7)$ & $92 / 197(46.7)$ & $1 / 13(7.7)$ \\
\hline Month 24 & $1279 / 1622(78.9)$ & $2608 / 2903(89.8)$ & ND & $8 / 13(61.5)$ \\
\hline Lumbar spine & $n 1=1750$ & $n 1=3170$ & $n 1=206$ & $n 1=16$ \\
\hline Month 12 & $1638 / 1722(95.1)$ & $3030 / 3151(96.2)$ & $179 / 197(90.9)$ & $9 / 13(69.2)$ \\
\hline Month 24 & $1479 / 1571(94.1)$ & $2797 / 2861(97.8)$ & ND & $13 / 13(100.0)$ \\
\hline \multicolumn{5}{|c|}{ Achieved BMD percentage change from baseline $\geq 6 \%$} \\
\hline Total hip & $n 1=1826$ & $n 1=3238$ & $n 1=206$ & $n 1=16$ \\
\hline Month 12 & $839 / 1781(47.1)$ & 1498/3197 (46.9) & 28/197 (14.2) & $0 / 13(0)$ \\
\hline Month 24 & $920 / 1622(56.7)$ & $2015 / 2903(69.4)$ & ND & $2 / 13(15.4)$ \\
\hline Lumbar spine & $n 1=1750$ & $n 1=3170$ & $n 1=206$ & $n 1=16$ \\
\hline Month 12 & $1513 / 1722(87.9)$ & 2812/3151 (89.2) & 147/197 (74.6) & $5 / 13(38.5)$ \\
\hline Month 24 & $1390 / 1571(88.5)$ & $2719 / 2861(95.0)$ & ND & $12 / 13(92.3)$ \\
\hline
\end{tabular}

Data analyzed were from four studies: (1) ARCH (NCT01631214), FRAME (NCT01575834), STRUCTURE (NCT01796301), and the Phase 2 extension (NCT00896532) using descriptive statistics

$n=$ number of patients who received romosozumab in each study. $n l=$ number of patients who received romosozumab in each study and had baseline and $\geq 1$ postbaseline assessment of the parameter of interest; $n 2=$ number of patients who achieved BMD percentage change from baseline $\geq 3 \%$ or $\geq 6 \% ; n 3=$ number of patients with evaluable data at the time of interest

${ }^{\text {a Month }} 12$ rows in the table are considering baseline to be the time when patients transitioned from denosumab to romosozumab (actually month 36 of the Phase 2 extension); month 24 rows in the table are considering baseline to be the time when patients transitioned from placebo to denosumab (actually month 24 of the Phase 2 extension). BMD bone mineral density, $N D$ not determined

romosozumab. Over 2 years, the proportions of patients who achieved BMD gains $\geq 3 \%$ and $\geq 6 \%$ increased to $79 \%$ and $57 \%$, respectively, with romosozumab followed by alendronate and were even higher for women who received romosozumab followed by denosumab (Table 2 ). In the Phase 2 extension, the responder rate for BMD gains $\geq 6 \%$ with denosumab followed by romosozumab was only $15 \%$.

\section{Lumbar spine BMD responder analysis}

More than $90 \%$ of participants had lumbar spine BMD gain $\geq 3 \%$ with 1 year of romosozumab in ARCH, FRAME, and STRUCTURE, whereas these responder rates were $88 \%, 89 \%$, and $75 \%$, respectively, for the $\geq 6 \%$ cutoff point (Table 2 ). In the Phase 2 extension, after completion of 1 year of denosumab treatment, the responder rates with 1 year of romosozumab were substantially lower for both cutoff points. Over 2 years, responder rates were very high for both $\geq 3 \%$ and $\geq 6 \%$ BMD gain with all treatment sequences (Table 2).

\section{Bone turnover markers: PINP and $\beta$-CTX}

Median serum changes in PINP and $\beta$-CTX levels across the four studies are shown in Fig. 3. During year 1 with romosozumab in ARCH, PINP increased by $42 \mu \mathrm{g} / \mathrm{L}$ (median) above baseline at the 1-month peak and decreased to below baseline after month 6 (Fig. 3a). Levels of $\beta$-CTX decreased by $55 \mathrm{ng} / \mathrm{L}$ below baseline at month 1 and remained below baseline throughout the 1 year of treatment (Fig. 3a). Levels of both PINP and $\beta$-CTX continued to decrease during year 2 upon transition to alendronate, with nadir levels at months 18 and 15 , respectively (Fig. 3a).

During year 1 with romosozumab in FRAME, changes in levels of PINP and $\beta$-CTX were similar to those seen in ARCH. PINP increased $44 \mu \mathrm{g} / \mathrm{L}$ above baseline at month 1 and then decreased to below baseline between months 6 and 9 (Fig. 3b). Levels of $\beta$-CTX decreased by $181 \mathrm{ng} / \mathrm{L}$ at month 1 and remained below baseline throughout the 1 year of treatment (Fig. 3b). Both PINP and $\beta$-CTX levels continued to decrease during year 2 upon transition to denosumab, with the nadir levels for both markers at month 18 (Fig. 3b). 


\section{a. ARCH (romosozumab before alendronate)}

\section{PINP}

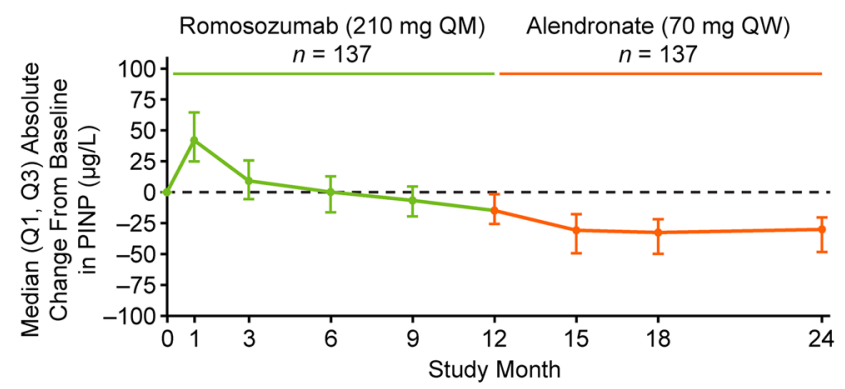

\section{b. FRAME (romosozumab before denosumab)}

\section{PINP}

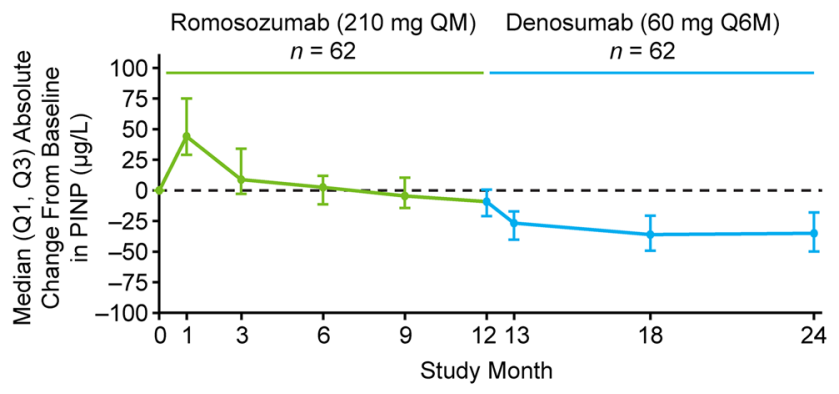

\section{c. STRUCTURE (romosozumab after alendronate)}

PINP

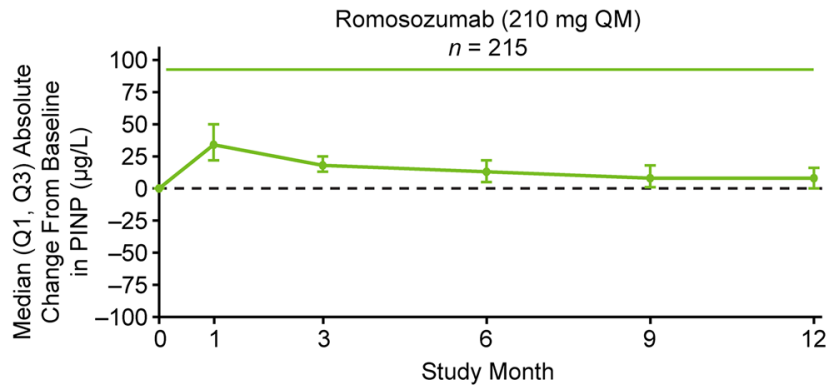

\section{$\beta-\mathrm{CTX}$}
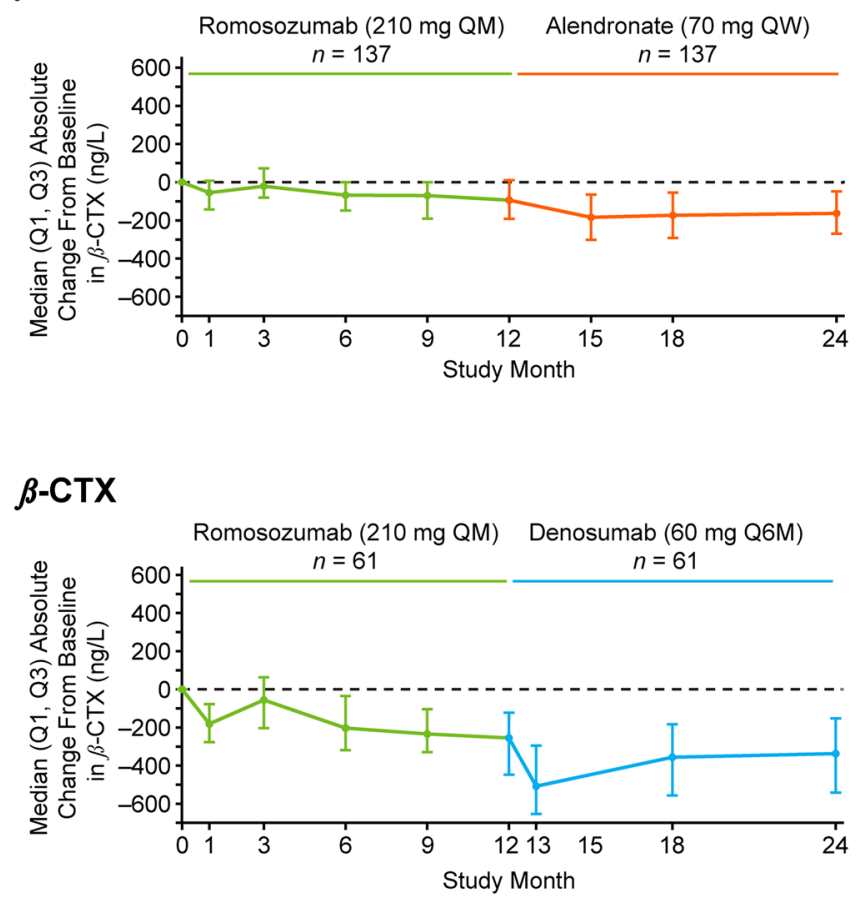

\section{d. Phase 2 Extension (romosozumab after denosumab)}

PINP

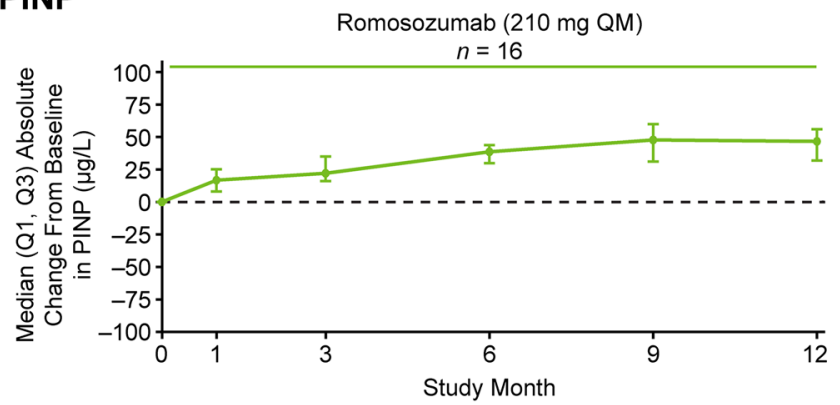

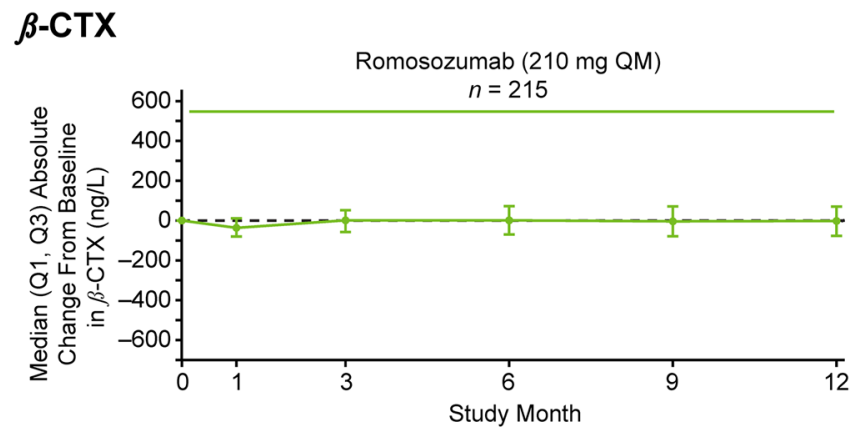

$\beta$-CTX

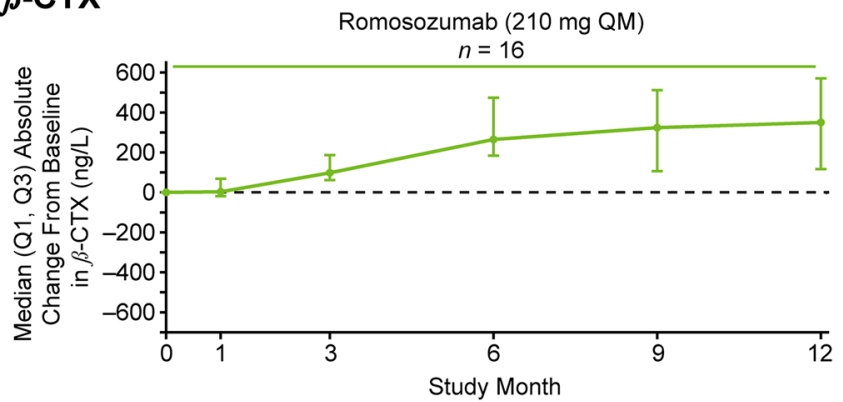

Fig. 3 Median absolute changes in serum PINP and $\beta$-CTX in women treated with romosozumab prior to antiresorptive therapy (a, b) and in women treated with romosozumab after alendronate (c) or denosumab (d). Data analyzed were from ARCH (NCT01631214), FRAME (NCT01575834), STRUCTURE (NCT01796301), and the Phase 2 extension (NCT00896532) using descriptive statistics. For the Phase 2 extension, baseline (month 0) is at month 36 of the study when patients transitioned from denosumab to romosozumab. $n=$ number of patients who received romosozumab in each study and had PINP and $\beta$-CTX assessments at baseline and specified timepoints. $\beta$-CTX $\beta$-isomer of the C-terminal telopeptide of type I collagen; $P I N P$ procollagen type 1 N-terminal propeptide; $Q 1, Q 3$ first and third quartiles; $Q 6 M$ every 6 months; $Q M$ monthly; $Q W$ weekly 
In STRUCTURE, after patients had switched from alendronate to romosozumab, serum PINP increased by $34 \mu \mathrm{g} / \mathrm{L}$ above baseline at month 1 and remained above baseline throughout the 1 year of treatment (Fig. 3c). Serum $\beta$-CTX was largely unchanged, decreasing slightly at month 1 and then returning to baseline for the remainder of the 1 year of treatment (Fig. 3c).

In the Phase 2 extension, after denosumab discontinuation and administration of romosozumab, changes in bone turnover markers followed a different pattern from those seen in the other three studies. PINP increased minimally at month 1, and PINP and $\beta$-CTX levels increased gradually, in parallel, with the peak PINP increment $(48 \mu \mathrm{g} / \mathrm{L}$ above baseline) at month 9 and peak $\beta$-CTX increment ( $350 \mathrm{ng} / \mathrm{L}$ above baseline) at month 12 (Fig. 3d).

\section{Discussion}

When used as initial therapy, 1 year of romosozumab produced large BMD gains at the total hip and lumbar spine, which appear greater than those seen with any other single therapy [32-34]. In women pretreated with alendronate, BMD gains with 1 year of romosozumab were lower than those in treatment-naïve women; approximately 50\% and $75 \%$ of the BMD increments achieved in treatment-naïve patients at the total hip and lumbar spine, respectively. These gains in total hip and lumbar spine BMD remain significant and were higher than those achieved upon transition from alendronate to teriparatide, as shown directly in STRUCTURE [12]. In women pretreated with denosumab, net BMD gains with 1 year of romosozumab were substantially lower than those in treatment-naïve women or those observed following transition from alendronate. However, stability of total hip BMD with romosozumab after denosumab compares favorably with transition to teriparatide in the DATA-Switch study (though not head-to-head data) [14], where hip BMD remained below the transition baseline, even after 2 years of teriparatide treatment. For the 2-year treatment sequences, large BMD gains at both the total hip and lumbar spine were observed when romosozumab was followed by denosumab or alendronate. BMD gains with the 2-year sequence of denosumab followed by romosozumab were comparably lower, with a more substantial differential effect on hip BMD. The 2-year responder analyses also indicate that a very high proportion of women will attain $\geq 6 \%$ BMD gain at the lumbar spine, regardless of treatment sequence. However, the sequence of romosozumab and denosumab had a greater differential impact on BMD response at the total hip.

Our results from the four RCTs reviewed are consistent with results observed in a prospective real-world study of patients treated with romosozumab for 12 months as first therapy or after receiving antiresorptive agents [13, 35]. In that study, BMD gains differed significantly in previously untreated patients, patients previously treated with bisphosphonates, or patients previously treated with denosumab; at the total hip, mean BMD gains were 5.6\%, 3.3\%, and 0.6\%, respectively, and at the lumbar spine, mean BMD gains were $18.2 \%, 10.2 \%$, and $6.4 \%$, respectively. Similar BMD gains at the total hip and lumbar spine were also reported in another prospective real-world study in which previously untreated patients and patients previously treated with bisphosphonates or denosumab were treated with romosozumab for 12 months [36] and in a retrospective real-world study in which previously untreated patients and patients previously treated with bisphosphonates were treated with romosozumab for 12 months [37].

We have limited data on the morphological basis of bone strength gains following antiresorptive or anabolic therapy. In the primary Phase 2 study, with romosozumab as initial therapy, quantitative computed tomography (QCT) assessments indicated that volumetric BMD and bone strength at the total hip increased $4.1 \%$ and $3.6 \%$, respectively, and these changes were a reflection of increments in both cortical and cancellous compartments; similarly, volumetric BMD and bone strength at the lumbar spine increased $17.7 \%$ and $27.3 \%$, respectively, with romosozumab, again with demonstrable increments in both cortical and cancellous compartments [38, 39]. In a subset of women from ARCH, QCT assessments of the lumbar spine also demonstrated increments in volumetric BMD and bone strength of both cortical and cancellous bone compartments [40]; however, there is no information about the cortical and cancellous bone compartments at the total hip since QCT assessments were not performed in the hip region in ARCH. In STRU CTURE, with romosozumab after alendronate, volumetric $\mathrm{BMD}$ and bone strength at the total hip increased 3.4\% and $2.5 \%$, respectively, with improvements in both cortical and cancellous bone compartments; however, gains in both compartments were diminished with the alendronate-toromosozumab sequence, compared with the romosozumab first sequence [12].

Despite the known limitations of interpreting bone turnover marker levels, absolute changes in serum levels of PINP and $\beta$-CTX across the studies reviewed here can help explain some of the BMD effects observed. In the four studies, serum PINP increments peaked at $34-48 \mu \mathrm{g} / \mathrm{L}$ above baseline with romosozumab and were similar across the studies; however, the kinetics of the PINP increase were different when romosozumab was administered after denosumab, where we observed a slower, progressive elevation and delayed peak. Moreover, the effects on $\beta$-CTX varied significantly based on whether patients were previously untreated, pretreated with alendronate, or pretreated with denosumab. Since most of the bone formation with romosozumab is modeling-based, 
and thus independent from baseline bone remodeling rate [41], we would expect minimal influence of previous antiresorptive treatment on PINP kinetics and BMD gain achieved with romosozumab as compared with teriparatide, where the majority of the bone formation is remodeling-based. Our findings are consistent with this thesis; however, some of the initial BMD gain might be related to overfilling of the remodeling cavities open at the time romosozumab is administered. The magnitude of bone remodeling surface is lower in patients who received prior antiresorptive agents compared with that in previously untreated patients [42-44], particularly with prior denosumab.

Consistent with the known dual effect of romosozumab, in previously untreated women, serum PINP levels increased and $\beta$-CTX levels declined in ARCH and FRAME. Serum $\beta$-CTX level decreased further upon transition to alendronate, again indicating that the antiresorptive potency of alendronate is greater than that of romosozumab. This has been demonstrated previously with direct comparisons of romosozumab and alendronate in the first year of the Phase 2 study [25] and in the first year of ARCH [5]. $\beta$-CTX levels declined to an even greater degree after transition from romosozumab to denosumab, consistent with the known greater antiresorptive potency of denosumab compared with alendronate [45-48]. The bigger differential effect between alendronate and denosumab (after romosozumab) on BMD gain at the hip, compared with the spine, might be related to differences in alendronate activity at these two skeletal sites. The effect of alendronate on remodeling suppression is near complete in cancellous bone of the spine, but much less prominent in cortical bone [48], leading to greater ultimate BMD gain at the total hip, with the sequence of romosozumab followed by denosumab in FRAME [27] versus romosozumab followed by alendronate in ARCH [5].

Following alendronate treatment [12], serum PINP increased on transition to romosozumab, while there was no significant change in $\beta$-CTX. This lack of change in $\beta$-CTX suggests that the antiresorptive effect of romosozumab is sufficiently potent to replace the partial loss of antiresorptive activity, superimposed on the residual alendronate action seen after alendronate withdrawal [49]. No further antiresorptive action is observed when romosozumab is substituted for alendronate. The most unusual profile of bone turnover marker changes with romosozumab was observed when administered after denosumab. The delayed increase in serum PINP suggests that ambient bone remodeling activity at the time of romosozumab initiation might contribute to romosozumab's early bone-forming effects. The progressive increase in $\beta$-CTX implies that the antiresorptive potency of romosozumab is insufficient to completely prevent the bone turnover rebound that normally occurs after denosumab cessation, suggesting that there is more bone resorbed over the year, certainly compared with that observed after transition from alendronate to romosozumab. Regardless, even after denosumab discontinuation, there is still a positive net balance between resorption and formation, as shown by the bone mass increase, albeit smaller than observed in treatment-naïve patients or when transitioning from alendronate. Our bone turnover marker results are also consistent with those observed in a prospective real-world study where the PINP response in previously untreated patients and in patients previously treated with bisphosphonates peaked at 1 month after transition to romosozumab, whereas the PINP response was delayed (peaked at 6 months) and prolonged in patients previously treated with denosumab after transition to romosozumab $[13,35]$.

For patients on bisphosphonates who still appear to be at high risk for fracture, switching to romosozumab leads to superior increases in BMD and bone strength than switching to teriparatide [12]. For patients on denosumab who have had a suboptimal response or who must discontinue treatment for other reasons [50], switching to romosozumab improves spine BMD and maintains or produces a small increment in hip BMD. In contrast, in the DATA-Switch study [14], women who switched from denosumab to teriparatide had a rapid decline in hip BMD, which persisted during 2 years of teriparatide treatment, and a large increase in serum $\beta$-CTX levels associated with both the effect of denosumab discontinuation and the pro-remodeling action of teriparatide [32, 51, 52]. In contrast to the transition from denosumab to teriparatide, the moderate intrinsic antiresorptive activity of romosozumab may contribute to a more favorable net balance between bone formation and resorption upon transition from denosumab. This might be of particular importance for patients sustaining a vertebral fracture despite denosumab therapy and for whom transition to romosozumab might be a preferred option. However, further research is needed to confirm the BMD effects with romosozumab after longer denosumab therapy and to confirm that this treatment sequence will be protective against occurrence of multiple vertebral fractures associated with denosumab withdrawal [53]. Although the transition from denosumab to romosozumab appears to be superior to the transition to teriparatide, this inference results from two separate studies with a limited number of participants and with no fracture endpoints; currently, there are no RCTs directly comparing these two regimens.

There are no fracture data from STRUCTURE and the Phase 2 extension where antiresorptives were given as the first treatment followed by romosozumab. We have drawn conclusions about the effectiveness of romosozumab treatment as first therapy based on mean BMD changes; BMD changes have been shown to relate to fracture resistance in multiple [15] but not all studies [54]. However, even in Reid et al. [54], where BMD gain was not associated with fracture risk, absolute BMD level attained after treatment was 
associated with fracture risk. The relationship between BMD attained on treatment and subsequent fracture rates has also been demonstrated in prior studies with zoledronic acid [18], alendronate [17, 20], denosumab [19], and romosozumab [20]. In this analysis, we are not comparing different treatments; our focus is on the effect of treatment sequence with romosozumab as the first therapy, compared with romosozumab as the second therapy. Our data show that greater improvement is likely when romosozumab is used first.

There are several limitations to the findings in our analysis. We summarized BMD data across four studies that had different populations with varying age, fracture prevalence, and baseline BMD; however, baseline BMD was adjusted within each trial to calculate BMD change (except in the Phase 2 extension due to small sample size). In contrast to the large sample sizes in ARCH, FRAME, and STRU CTURE, for the Phase 2 extension, only 16 patients were evaluated. Interestingly, the findings discussed here are very similar to those observed in another small arm of the Phase 2 extension when patients received a second course of romosozumab after an intervening year of denosumab [28]. A very important caveat is that the effects of switching from denosumab to romosozumab were investigated after only 1 year of denosumab. In contrast, in STRUCTU $\mathrm{RE}$ [12], women had been on bisphosphonates for more than 6 years (median treatment) before transition to romosozumab. Clearly, more research is needed to evaluate the safety and efficacy of longer pretreatment with denosumab prior to romosozumab [55]. Data are not available for the first year of the alendronate-to-romosozumab sequence because BMD increments on alendronate in STRUCTURE [12] were not determined (as the study was not designed to answer this question). We also acknowledge that the changes in bone turnover markers may not necessarily reflect what is occurring at the bone surface level in different skeletal sites and that the BMD changes are influenced by both change in mass and mineralization. The results here are entirely descriptive without formal statistical analyses conducted. Finally, we recognize that in some regions, financial considerations require initial antiresorptive therapy rather than the more expensive bone anabolic therapy. It is imperative that clinicians understand the differential bone effects of bisphosphonates versus denosumab as initial therapy before transitioning to romosozumab in the clinical management of their patients. The strengths of this analysis include the robustness of the studies conducted [5, 12, 27-29], with frequent quality measurements of both bone turnover markers and BMD and the power to detect significant changes in the endpoints considered. Our findings are consistent with those from similar groups of patients in real-world observational studies [13, 35-37].

In conclusion, results from our study show that initial treatment with 1 year of romosozumab produces substantial BMD gains at the total hip and lumbar spine, and subsequent transition from romosozumab to a potent antiresorptive augments those gains. Romosozumab also effectively increases both hip and spine BMD after alendronate and may improve or at least maintain BMD in patients after short-term denosumab (1 year). Since BMD on treatment is a strong surrogate for fracture reduction [17-20], these findings support the concept that untreated patients at high risk of fracture should be considered for initial treatment with romosozumab. Our results are concordant with recent estimates that a sequence of boneforming agents followed by antiresorptive agents would prevent more fractures than the reverse sequence [56]. Since osteoporosis is a chronic condition requiring longterm therapy, for those patients at very high risk for fracture who will likely need both bone-forming and antiresorptive agents, improved clinical efficacy will be seen if the bone-forming agent is used as initial therapy.

Supplementary Information The online version contains supplementary material available at https://doi.org/10.1007/s00198-021-06174-0.

Acknowledgements Lisa A. Humphries, $\mathrm{PhD}$, of Amgen Inc. and Martha Mutomba (on behalf of Amgen Inc.) provided medical writing support.

Author contributions The first author (FC) proposed the study, developed the initial analysis plan, had full access to the data, and wrote the first draft and extensively revised subsequent drafts of the manuscript. Amgen Inc. maintained the study database and performed the analyses. The first author (FC) and 2 authors from Amgen Inc. (MKO and CEM) take responsibility for the integrity of the data analysis. All authors participated in the analysis and/or interpretation of the data and participated in the critical review and revision of the manuscript drafts. All authors approved the final version for submission.

Funding Amgen Inc. and UCB Pharma sponsored this study.

Data availability Qualified researchers may request data from Amgen clinical studies. Complete details are available at the following: https:// wwwext.amgen.com/science/clinical-trials/clinical-data-transparencypractices/clinical-trial-data-sharing-request/.

Code availability Not applicable.

\section{Declarations}

Ethics approval Not applicable.

Consent to participate Not applicable.

Consent for publication Not applicable.

Conflicts of interest FC has received institutional grants and research support from Amgen, has served as a consultant for Amgen and Radius Health, and has served on the speakers' bureaus for Amgen and Radius Health. DLK has received institutional grants and research support from Amgen and Radius Health; has served as a consultant for Amgen, Eli Lilly, and Pfizer; and has served on the speakers' bu- 
reaus for Amgen and Eli Lilly. BLL has received institutional grants and research support from Amgen and Novo Nordisk; has served as a consultant for Amgen, UCB Pharma, Eli Lilly, Gedeon Richter, and Gilead; and has served on the speakers' bureaus for Eli Lilly, Amgen, and UCB Pharma. BZL has received institutional grants and research support from Amgen and has served as a consultant for Amgen and Radius Health. EML has received research grants from Radius Health, Amgen, Mereo, and Bindex; income for service on scientific advisory boards or consulting for Amgen, Radius, Alexion, Sandoz, Samsung Bioepis, and Sanifit; income for service on speakers' bureaus for Radius and Alexion; project development support for University of New Mexico; and royalties from UpToDate for sections on DXA, fracture risk assessment, and prevention of osteoporosis. He is a board member of the National Osteoporosis Foundation, International Society for Clinical Densitometry, and Osteoporosis Foundation of New Mexico. AM has served as a consultant for Amgen, Amgen Astellas BioPharma K.K., and Teijin Pharma. MR, MM, MKO, and CEM are employees and stockholders of Amgen. CL is an employee and stockholder of UCB Pharma. SF has received institutional grants and research support from Amgen, UCB Pharma, AgNovos, Alexion, and Labatec and has served as a consultant for Amgen, UCB Pharma, Alexion, AgNovos, Radius Health, and Labatec.

Open Access This article is licensed under a Creative Commons Attribution-NonCommercial 4.0 International License, which permits any non-commercial use, sharing, adaptation, distribution and reproduction in any medium or format, as long as you give appropriate credit to the original author(s) and the source, provide a link to the Creative Commons licence, and indicate if changes were made. The images or other third party material in this article are included in the article's Creative Commons licence, unless indicated otherwise in a credit line to the material. If material is not included in the article's Creative Commons licence and your intended use is not permitted by statutory regulation or exceeds the permitted use, you will need to obtain permission directly from the copyright holder. To view a copy of this licence, visit http://creativecommons.org/licenses/by-nc/4.0/.

\section{References}

1. Saag KG, Shane E, Boonen S, Marín F, Donley DW, Taylor KA, Dalsky GP, Marcus R (2007) Teriparatide or alendronate in glucocorticoid-induced osteoporosis. N Engl J Med 357:2028-2039

2. Saag KG, Zanchetta JR, Devogelaer J-P, Adler RA, Eastell R, See K, Krege JH, Krohn K, Warner MR (2009) Effects of teriparatide versus alendronate for treating glucocorticoid-induced osteoporosis: thirty-six-month results of a randomized, double-blind, controlled trial. Arthritis Rheum 60:3346-3355

3. Hadji P, Zanchetta JR, Russo L, Recknor CP, Saag KG, McKiernan FE, Silverman SL, Alam J, Burge RT, Krege JH, Lakshmanan MC, Masica DN, Mitlak BH, Stock JL (2012) The effect of teriparatide compared with risedronate on reduction of back pain in postmenopausal women with osteoporotic vertebral fractures. Osteoporos Int 23:2141-2150

4. Kendler DL, Marin F, Zerbini CAF, Russo LA, Greenspan SL, Zikan V, Bagur A, Malouf-Sierra J, Lakatos P, Fahrleitner-Pammer A, Lespessailles E, Minisola S, Body JJ, Geusens P, Möricke R, López-Romero P (2018) Effects of teriparatide and risedronate on new fractures in post-menopausal women with severe osteoporosis (VERO): a multicentre, double-blind, double-dummy, randomised controlled trial. Lancet 391:230-240
5. Saag KG, Petersen J, Brandi ML, Karaplis AC, Lorentzon M, Thomas T, Maddox J, Fan M, Meisner PD, Grauer A (2017) Romosozumab or alendronate for fracture prevention in women with osteoporosis. N Engl J Med 377:1417-1427

6. Camacho PM, Petak SM, Binkley N, Diab DL, Eldeiry LS, Farooki A, Harris ST, Hurley DL, Kelly J, Lewiecki EM, Pessah-Pollack R, McClung M, Wimalawansa SJ, Watts NB (2020) American Association of Clinical Endocrinologists/American College of Endocrinology clinical practice guidelines for the diagnosis and treatment of postmenopausal osteoporosis-2020 update executive summary. Endocr Pract 26:564-570

7. Cosman F (2020) Anabolic therapy and optimal treatment sequences for patients with osteoporosis at high risk for fracture. Endocr Pract 26:777-786

8. Ettinger B, Martin SJ, Crans G, Pavo I (2004) Differential effects of teriparatide on BMD after treatment with raloxifene or alendronate. J Bone Miner Res 19:745-751

9. Boonen S, Marin F, Obermayer-Pietsch B, Simões ME, Barker C, Glass EV, Hadji P, Lyritis G, Oertel H, Nickelsen T, McCloskey EV, for the EUROFORS Investigators, (2008) Effects of previous antiresorptive therapy on the bone mineral density response to two years of teriparatide treatment in postmenopausal women with osteoporosis. J Clin Endocrinol Metab 93:852-860

10. Miller PD, Delmas PD, Lindsay R, Watts NB, Luckey M, Adachi J, Saag K, Greenspan SL, Seeman E, Boonen S, Meeves S, Lang TF, Bilezikian JP (2008) Early responsiveness of women with osteoporosis to teriparatide after therapy with alendronate or risedronate. J Clin Endocrinol Metab 93:3785-3793

11. Cosman F, Wermers RA, Recknor C, Mauck KF, Xie L, Glass EV, Krege JH (2009) Effects of teriparatide in postmenopausal women with osteoporosis on prior alendronate or raloxifene: differences between stopping and continuing the antiresorptive agent. J Clin Endocrinol Metab 94:3772-3780

12. Langdahl BL, Libanati C, Crittenden DB, Bolognese MA, Brown JP, Daizadeh NS, Dokoupilova E, Engelke K, Finkelstein JS, Genant HK, Goemaere S, Hyldstrup L, Jodar-Gimeno E, Keaveny TM, Kendler D, Lakatos P, Maddox J, Malouf J, Massari FE, Molina JF, Ulla MR, Grauer A (2017) Romosozumab (sclerostin monoclonal antibody) versus teriparatide in postmenopausal women with osteoporosis transitioning from oral bisphosphonate therapy: a randomised, open-label, phase 3 trial. Lancet 390:1585-1594

13. Ebina K, Hirao M, Tsuboi H, Nagayama Y, Kashii M, Kaneshiro S, Miyama A, Nakaya H, Kunugiza Y, Okamura G, Etani Y, Takami K, Goshima A, Nakata K (2020) Effects of prior osteoporosis treatment on early treatment response of romosozumab in patients with postmenopausal osteoporosis. Bone 140:115574

14. Leder BZ, Tsai JN, Uihlein AV, Wallace PM, Lee H, Neer RM, Burnett-Bowie S-AM (2015) Denosumab and teriparatide transitions in postmenopausal osteoporosis (the DATA-Switch study): extension of a randomised controlled trial. Lancet 386:1147-1155

15. Bouxsein ML, Eastell R, Lui L-Y, Wu LA, de Papp AE, Grauer A, Marin F, Cauley JA, Bauer DC, Black DM, FNIH Bone Quality Project (2019) Change in bone density and reduction in fracture risk: a meta-regression of published trials. J Bone Miner Res 34:632-642

16. Cummings SR, Cosman F, Eastell R, Reid IR, Mehta M, Lewiecki EM (2013) Goal-directed treatment of osteoporosis. J Bone Miner Res 28:433-438

17. Black DM, Schwartz AV, Ensrud KE, Cauley JA, Levis S, Quandt SA, Satterfield S, Wallace RB, Bauer DC, Palermo L, Wehren LE, Lombardi A, Santora AC, Cummings SR, for the FLEX Research Group (2006) Effects of continuing or stopping alendronate after 5 years of treatment: the Fracture Intervention Trial Long-term Extension (FLEX): a randomized trial. JAMA 296:2927-2938

18. Cosman F, Cauley JA, Eastell R, Boonen S, Palermo L, Reid IR, Cummings SR, Black DM (2014) Reassessment of fracture risk 
in women after 3 years of treatment with zoledronic acid: when is it reasonable to discontinue treatment? J Clin Endocrinol Metab 99:4546-4554

19. Ferrari S, Libanati C, Lin CJF, Brown JP, Cosman F, Czerwiński E, de Gregório LH, Malouf-Sierra J, Reginster J-Y, Wang A, Wagman RB, Lewiecki EM (2019) Relationship between bone mineral density T-score and nonvertebral fracture risk over 10 years of denosumab treatment. J Bone Miner Res 34:1033-1040

20. Cosman F, Lewiecki EM, Ebeling PR, Hesse E, Napoli N, Matsumoto T, Crittenden DB, Rojeski M, Yang W, Libanati C, Ferrari $\mathrm{S}$ (2020) T-score as an indicator of fracture risk during treatment with romosozumab or alendronate in the ARCH trial. J Bone Miner Res 35:1333-1342

21. Cosman F, Nieves JW, Dempster DW (2017) Treatment sequence matters: anabolic and antiresorptive therapy for osteoporosis. J Bone Miner Res 32:198-202

22. Cosman F, Miller PD, Williams GC, Hattersley G, Hu M-Y, Valter I, Fitzpatrick LA, Riis BJ, Christiansen C, Bilezikian JP, Black D (2017) Eighteen months of treatment with subcutaneous abaloparatide followed by 6 months of treatment with alendronate in postmenopausal women with osteoporosis: results of the ACTIVExtend trial. Mayo Clin Proc 92:200-210

23. Bone HG, Cosman F, Miller PD, Williams GC, Hattersley G, Hu MY, Fitzpatrick LA, Mitlak B, Papapoulos S, Rizzoli R, Dore RK, Bilezikian JP, Saag KG (2018) ACTIVExtend: 24 months of alendronate after 18 months of abaloparatide or placebo for postmenopausal osteoporosis. J Clin Endocrinol Metab 103:2949-2957

24. EVENITY $^{\circledR}$ (romosozumab-aqqg) US prescribing information (2019) Amgen Inc., Thousand Oaks, CA, USA. https://www.pi. amgen.com/ /media/amgen/repositorysites/pi-amgen-com/eveni ty/evenity_pi_hcp_english.ashx. Accessed 21 Sept 2021

25. McClung MR, Grauer A, Boonen S, Bolognese MA, Brown JP, Diez-Perez A, Langdahl BL, Reginster J-Y, Zanchetta JR, Wasserman SM, Katz L, Maddox J, Yang Y-C, Libanati C, Bone HG (2014) Romosozumab in postmenopausal women with low bone mineral density. N Engl J Med 370:412-420

26. Padhi D, Jang G, Stouch B, Fang L, Posvar E (2011) Single-dose, placebo-controlled, randomized study of AMG 785, a sclerostin monoclonal antibody. J Bone Miner Res 26:19-26

27. Cosman F, Crittenden DB, Adachi JD, Binkley N, Czerwinski E, Ferrari S, Hofbauer LC, Lau E, Lewiecki EM, Miyauchi A, Zerbini CA, Milmont CE, Chen L, Maddox J, Meisner PD, Libanati C, Grauer A (2016) Romosozumab treatment in postmenopausal women with osteoporosis. N Engl J Med 375:1532-1543

28. Kendler DL, Bone HG, Massari F, Gielen E, Palacios S, Maddox J, Yan C, Yue S, Dinavahi RV, Libanati C, Grauer A (2019) Bone mineral density gains with a second 12-month course of romosozumab therapy following placebo or denosumab. Osteoporos Int 30:2437-2448

29. McClung MR, Bolognese MA, Brown JP, Reginster J-Y, Langdahl BL, Shi Y, Timoshanko J, Libanati C, Chines A, Oates MK (2021) Skeletal responses to romosozumab after 12 months of denosumab. JBMR Plus 5:e10512

30. McClung MR, Brown JP, Diez-Perez A, Resch H, Caminis J, Meisner P, Bolognese MA, Goemaere S, Bone HG, Zanchetta JR, Maddox J, Bray S, Grauer A (2018) Effects of 24 months of treatment with romosozumab followed by 12 months of denosumab or placebo in postmenopausal women with low bone mineral density: a randomized, double-blind, phase 2, parallel group study. J Bone Miner Res 33:1397-1406

31. Cosman F, Crittenden DB, Ferrari S, Khan A, Lane NE, Lippuner K, Matsumoto T, Milmont CE, Libanati C, Grauer A (2018) FRAME study: the foundation effect of building bone with 1 year of romosozumab leads to continued lower fracture risk after transition to denosumab. J Bone Miner Res 33:1219-1226
32. Neer RM, Arnaud CD, Zanchetta JR, Prince R, Gaich GA, Reginster JY, Hodsman AB, Eriksen EF, Ish-Shalom S, Genant HK, Wang O, Mitlak BH (2001) Effect of parathyroid hormone (1-34) on fractures and bone mineral density in postmenopausal women with osteoporosis. N Engl J Med 344:1434-1441

33. Cummings SR, San Martin J, McClung MR, Siris ES, Eastell R, Reid IR, Delmas P, Zoog HB, Austin M, Wang A, Kutilek S, Adami S, Zanchetta J, Libanati C, Siddhanti S, Christiansen C, Trial Collaborators FREEDOM (2009) Denosumab for prevention of fractures in postmenopausal women with osteoporosis. N Engl J Med 361:756-765

34. Black DM, Delmas PD, Eastell R, Reid IR, Boonen S, Cauley JA, Cosman F, Lakatos P, Leung PC, Man Z, Mautalen C, Mesenbrink P, Hu H, Caminis J, Tong K, Rosario-Jansen T, Krasnow J, Hue TF, Sellmeyer D, Eriksen EF, Cummings SR (2007) Once-yearly zoledronic acid for treatment of postmenopausal osteoporosis. N Engl J Med 356:1809-1822

35. Ebina K, Tsuboi H, Nagayama Y, Kashii M, Kaneshiro S, Miyama A, Nakaya H, Kunugiza Y, Hirao M, Okamura G, Etani Y, Takami K, Goshima A, Miura T, Nakata K, Okada S (2021) Effects of prior osteoporosis treatment on 12-month treatment response of romosozumab in patients with postmenopausal osteoporosis. Joint Bone Spine 88:105219

36. Kobayakawa T, Suzuki T, Nakano M, Saito M, Miyazaki A, Takahashi J, Nakamura Y (2021) Real-world effects and adverse events of romosozumab in Japanese osteoporotic patients: a prospective cohort study. Bone Rep 14:101068

37. Tominaga A, Wada K, Okazaki K, Nishi H, Terayama Y, Kato Y (2021) Early clinical effects, safety, and predictors of the effects of romosozumab treatment in osteoporosis patients: one-year study. Osteoporos Int 32:1999-2009

38. Keaveny TM, Crittenden DB, Bolognese MA, Genant HK, Engelke K, Oliveri B, Brown JP, Langdahl BL, Yan C, Grauer A, Libanati C (2017) Greater gains in spine and hip strength for romosozumab compared with teriparatide in postmenopausal women with low bone mass. J Bone Miner Res 32:1956-1962

39. Genant HK, Engelke K, Bolognese MA, Mautalen C, Brown JP, Recknor C, Goemaere S, Fuerst T, Yang Y-C, Grauer A, Libanati C (2017) Effects of romosozumab compared with teriparatide on bone density and mass at the spine and hip in postmenopausal women with low bone mass. J Bone Miner Res 32:181-187

40. Brown JP, Engelke K, Keaveny TM, Chines A, Chapurlat R, Foldes AJ, Nogues X, Civitelli R, De Villiers T, Massari F, Zerbini CAF, Wang Z, Oates MK, Recknor C, Libanati C (2021) Romosozumab improves lumbar spine bone mass and bone strength parameters relative to alendronate in postmenopausal women: results from the Active-Controlled Fracture Study in Postmenopausal Women With Osteoporosis at High Risk (ARCH) trial. J Bone Miner Res

41. Chavassieux P, Chapurlat R, Portero-Muzy N, Roux J-P, Garcia P, Brown JP, Libanati C, Boyce RW, Wang A, Grauer A (2019) Bone-forming and antiresorptive effects of romosozumab in postmenopausal women with osteoporosis: bone histomorphometry and microcomputed tomography analysis after 2 and 12 months of treatment. J Bone Miner Res 34:1597-1608

42. Chavassieux PM, Arlot ME, Reda C, Wei L, Yates AJ, Meunier PJ (1997) Histomorphometric assessment of the long-term effects of alendronate on bone quality and remodeling in patients with osteoporosis. J Clin Invest 100:1475-1480

43. Dempster DW, Brown JP, Fahrleitner-Pammer A, Kendler D, Rizzo S, Valter I, Wagman RB, Yin X, Yue SV, Boivin G (2018) Effects of long-term denosumab on bone histomorphometry and mineralization in women with postmenopausal osteoporosis. J Clin Endocrinol Metab 103:2498-2509 
44. Reid IR, Miller PD, Brown JP, Kendler DL, Fahrleitner-Pammer A, Valter I, Maasalu K, Bolognese MA, Woodson G, Bone H, Ding B, Wagman RB, San Martin J, Ominsky MS, Dempster DW (2010) Effects of denosumab on bone histomorphometry: the FREEDOM and STAND studies. J Bone Miner Res 25:2256-2265

45. Lin T, Wang C, Cai X-Z, Zhao X, Shi M-M, Ying Z-M, Yuan F-Z, Guo C, Yan S-G (2012) Comparison of clinical efficacy and safety between denosumab and alendronate in postmenopausal women with osteoporosis: a meta-analysis. Int J Clin Pract 66:399-408

46. Kendler DL, Roux C, Benhamou CL, Brown JP, Lillestol M, Siddhanti S, Man H-S, San Martin J, Bone HG (2010) Effects of denosumab on bone mineral density and bone turnover in postmenopausal women transitioning from alendronate therapy. J Bone Miner Res 25:72-81

47. Brown JP, Prince RL, Deal C, Recker RR, Kiel DP, de Gregorio LH, Hadji P, Hofbauer LC, Alvaro-Gracia JM, Wang H, Austin M, Wagman RB, Newmark R, Libanati C, San Martin J, Bone HG (2009) Comparison of the effect of denosumab and alendronate on BMD and biochemical markers of bone turnover in postmenopausal women with low bone mass: a randomized, blinded, phase 3 trial. J Bone Miner Res 24:153-161

48. Seeman E, Delmas PD, Hanley DA, Sellmeyer D, Cheung AM, Shane E, Kearns A, Thomas T, Boyd SK, Boutroy S, Bogado C, Majumdar S, Fan M, Libanati C, Zanchetta J (2010) Microarchitectural deterioration of cortical and trabecular bone: differing effects of denosumab and alendronate. J Bone Miner Res 25:1886-1894

49. Kim TY, Bauer DC, McNabb BL, Schafer AL, Cosman F, Black DM, Eastell R (2019) Comparison of BMD changes and bone formation marker levels 3 years after bisphosphonate discontinuation: FLEX and HORIZON-PFT extension I trials. J Bone Miner Res 34:810-816

50. Kondo H, Okimoto N, Yoshioka T, Akahoshi S, Fuse Y, Ogawa T, Okazaki Y, Katae Y, Tsukamoto M, Yamanaka Y, Kawasaki
M, Sakai A (2020) Zoledronic acid sequential therapy could avoid disadvantages due to the discontinuation of less than 3-year denosumab treatment. J Bone Miner Metab 38:894-902

51. Miller PD, Hattersley G, Riis BJ, Williams GC, Lau E, Russo LA, Alexandersen P, Zerbini CAF, Hu MY, Harris AG, Fitzpatrick LA, Cosman F, Christiansen C, Active Study Investigators (2016) Effect of abaloparatide vs placebo on new vertebral fractures in postmenopausal women with osteoporosis: a randomized clinical trial. JAMA 316:722-733

52. Cosman F, McMahon D, Dempster D, Nieves JW (2020) Standard versus cyclic teriparatide and denosumab treatment for osteoporosis: a randomized trial. J Bone Miner Res 35:219-225

53. Kashii M, Ebina K, Kitaguchi K, Yoshikawa H (2020) Romosozumab was not effective in preventing multiple spontaneous clinical vertebral fractures after denosumab discontinuation: a case report. Bone Rep 13:100288

54. Reid IR, Horne AM, Mihov B, Stewart A, Bolland MJ, Bastin S, Gamble GD (2021) Predictors of fracture in older women with osteopenic hip bone mineral density treated with zoledronate. $\mathrm{J}$ Bone Miner Res 36:61-66

55. Sølling AS, Harsløf T, Langdahl B (2020) Treatment with zoledronate subsequent to denosumab in osteoporosis: a randomized trial. J Bone Miner Res 35:1858-1870

56. Kanis JA, Harvey NC, McCloskey E, Bruyère O, Veronese N, Lorentzon M, Cooper C, Rizzoli R, Adib G, Al-Daghri N, Campusano C, Chandran M, Dawson-Hughes B, Javaid K, Jiwa F, Johansson H, Lee JK, Liu E, Messina D, Mkinsi O, Pinto D, Prieto-Alhambra D, Saag K, Xia W, Zakraoui L, Reginster J-Y (2020) Algorithm for the management of patients at low, high and very high risk of osteoporotic fractures. Osteoporos Int 31:1-12

Publisher's note Springer Nature remains neutral with regard to jurisdictional claims in published maps and institutional affiliations. 